\title{
The Incompletely Liquid-Filled Two-Phase Numerical Simulation and Experimental Research of the of Fluid Couplings
}

\author{
Lidan Fan ${ }^{1, a}$ \\ ${ }^{1}$ College of Mechanical Engineering Jilin Engineering Normal University, Changchun, China \\ a574024123@qq.com
}

\begin{abstract}
Keywords: fluid couplings; gas-liquid two-phase flow; PIV test technology; CFD simulation.
Abstract. CFD simulation method and PIV technique provide efficient methods to reveal the highly complicated internal flow situation of fluid couplings. Although there are quite a number of papers adopting these two methods to analyze the internal flow situation of fluid coupling, most of which only consider single-phase flow. However, in the practical works, the inner fluid field of fluid coupling always refers to two-phase flow both for liqud and gas. In order to indicate the complicated mechanism of coupling internal gas-liquid two- phase flow state more authentically, this thesis simulates the flow field of the two typic fluid couplings work conditions by computational fluid dynamics (CFD) software and adopts the two-dimensional PIV technique of modern testing technology to measure and analyze it. Through the combination of experimental and numerical simulation, it probes into the characteristics of flow field when fluid couplings is under different liquid-filled rate.
\end{abstract}

\section{Introduction}

The fluid couplings are widely used in metallurgy, petroleum, mining, chemical industry, power generation and other areas. It makes the machines have the advantages of energy saving, vibration impact absorbing, easy starting and overload protecting and so on. In most conditions, the cyclic flow in the fluid couplings is the two-phase melding flow of air and hydraulic transmission oil. Even the full-filled fluid couplings are not completely liquid filled in order to leave the hydraulic transmission oil some space for expansion when it heated ${ }^{[1]}$. (see Bai 1997). Therefore, there is more practical significance to research the work condition of gas-liquid two-phase flow of the fluid coupling and its impact on its performance.

The internal flow of the fluid couplings is unsteady three dimensional flow. Besides, the viscosity and incompressible feature of the flow medium makes the flow field so complicated that it is always difficult to observe and accurate the flow. Because knowing the internal flow situationn and function rules are crucial to improve the performance of the fluid coupling, overseas researchers adopt various methods to analyze the internal flow of fliud coupling. Experimental research and the numerical simulation method are currently two basic ways to analyze the fluid motion law, which are mutually dependent on and promoted by each other. It is necessary to combine the two ways to solve the practical problems.

Until now, PIV technique is considered to be the most effective way to measure the internal flow field of turbomachine ${ }^{[2]}$ (see Choi et al. 2004). A few years ago, some researchers got the velocity vector images of turbomachine flow field by PIV technique, like Paone et $\mathrm{al}^{[3]}$.(1988), Hayami et $\mathrm{al}^{[4]}$.(1997) and Sinha et $\mathrm{al}^{[5]}(2000)$. But there still are many problems when use PIV technique to measure the gas-liquid two-phase flow, such as how to distinguish bubbles and tracer particles, how to remove the scatter light of bubbles and so on (see Sakakibara et al.2007). Fujiwara et al.(2004) and Lindken et al.(2002) had measured the gas-liquid two-phase flow with simple models. Until now, nobody has got the information of the gas-liquid two-phase flow in fluid couplings through PIV technique.

Though PIV technique can acquire relatively accurate information on the flow speed of complicated flow field, it can't offer the internal features of the flow field, such as the distribution of pressure and the distribution of phase volume. While the CFD numerical simulation can make upor 
this shortages. L. Bai(1997)，Charles N. McKinnon（2001），H. Huitenga and N. K. Mitra (2000) etc .had adopted the method of CFD numerical simulation to simulate the flow field of the full-filled fluid couplings and the results they got played an important role in improving the structure of fluid couplings. The reserches mentioned above are all based on the conditon when fluid coupling are full-filled. While the research for the flow field of normally working incompletely filled variable speed fluid coupling is seldom neither at home nor abord. Chunbao Liu (2008), Yandong He (2008) etc. have done a lot of work in this area and given the direction of future research.

In order to make the research getting closer to the actual working conditions of fluid couplings, this paper will research the internal flow field of the fluid couplings under the same rotate speed but different working conditions and liquid-filled ratio. Make use of both the PIV technique and the CFD numerical simulation methods to research the motion law of internal gas-liquid two-phase flow in fluid couplings. Through the comparative analysis on simulation and experimental results, it can make a more accurate and comprehensive analysis on the flow field of fluid coupling.

\section{CFD NUMERICAL SIMULATION}

Governing Equations. When luqid flow in internal fluid couplings is a continuous, three-dimensional, viscous and two-phase flow, the equation is:

When luqid flow in internal fluid couplings is a continuous, three-dimensional, viscous and two-phase flow, the equation is:

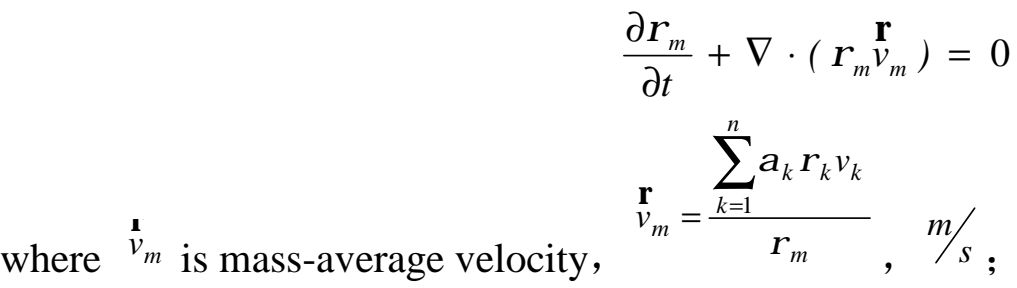

$$
\begin{aligned}
& \rho_{m} \text { is mixture densities, } \rho_{m}=\sum_{k=1}^{n} \alpha_{k} \rho_{k}, \mathrm{~kg} / \mathrm{m}^{3} ; \\
& \alpha_{k} \text { is the volume fraction of } k \text { phase; }
\end{aligned}
$$

$\rho_{k}$ is the densities of $k$ phase, $\mathrm{kg} / \mathrm{m}^{3}$ 。

Momentum equation can be acquired by summing all the momentum equation of respective phase. It can be expressed as

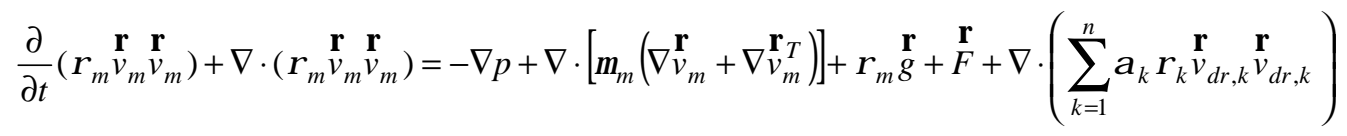

where $n$ is phase number;

$F$ is volumetric force, $N$;

$\nabla$ is Hamilton operator;

$$
\begin{aligned}
& \mu_{m} \text { is mixed viscosity, } \mu_{m}=\sum_{k=1}^{n} \alpha_{k} \mu_{k}, \quad p a \cdot s ; \\
& v_{d r, k} \text { is the drift velocity of phase } k, \quad \dot{v}_{d r, k}=v_{k}-v_{m}, \mathrm{~m} / \mathrm{s} \text { 。 }
\end{aligned}
$$

The equation of volume fraction of phase ${ }^{p}$ 


$$
\frac{\partial}{\partial t}\left(\alpha_{p} \rho_{p}\right)+\nabla \cdot\left(\alpha_{p} \rho_{p} v_{m}\right)=-\nabla \cdot\left(\alpha_{p} \rho_{p} \underset{v_{d r, p}}{r}\right)
$$

where $\alpha_{p}$ is the volume fraction of phase $^{p}$;

$$
\begin{aligned}
& \rho_{p} \text { is the densities of phase } p, \quad \mathrm{~kg} / \mathrm{m}^{3} \text {; }
\end{aligned}
$$

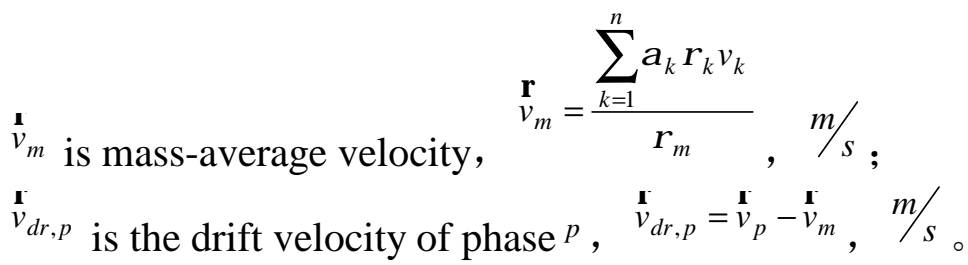

Model installment. The model is set up according the lucite model of fluid couplings in PIV expriment. The effctive diameter of the circle of circulation is $\mathrm{D}=230 \mathrm{~mm}$ and blades thickness is $4 \mathrm{~mm}$. Figure 1(a) is three-dimensional modle of fluid couplings. In order to get more accurately calculation, overall flow channel is adpoted to analyze. The function flow channel of fluid couplings is formed by the space between the case bodies of both impeller and turbine and blades.(figure1(b)). Because the case bodies are not involving in counting, they can be ignored.

Grid model is geometry expression of CFD modle as well as the carrier of counting and analyszing. In order to get the grid model of flow pass, we first get the three-dimensional flow pass model from the impeller UG model and then lead that model in Gambit to divide grid, forming grid mode of flow pass for calculating. (figure 1(c)) The inside flow of fluid coupling is very unstable and there is strong interaction between the flow channel of pump and turbine. In order to make the unitive calculation, sliding grid theory can be used to count the interface of the flow passes of pump and turbine. The paper regards the faying surface of pump and turbine as interface. Other surface is regarded as wall. After finished the settlement of the model, respectively do the calculation of the braking condition ( $i=0$ ) and rated condition( $i=0.97$ ) when the imput rotate speed is $n_{B}=160 \mathrm{r} / \mathrm{min}$ while the liquid-filled rate are $35 \%$ and $75 \%$.

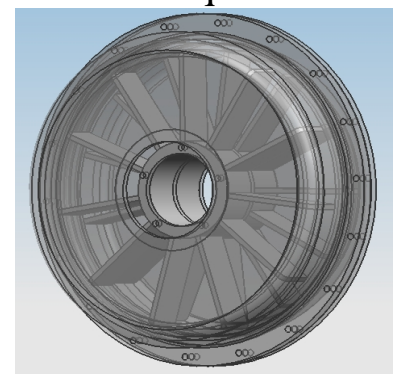

(a)three-dimensional modle

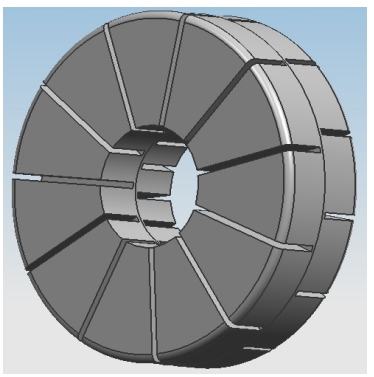

(b) flow channel model

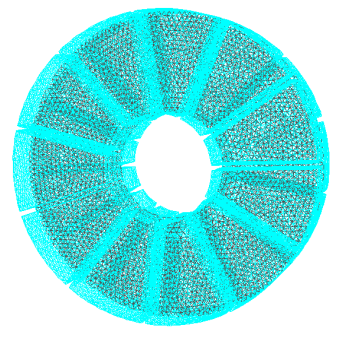

(c) grid model

Figure1.fluid couplings model

\section{Analysis of flow field}

Braking condition.Fig. 2 is the velocity vectors contour of flow field in pump and turbine when liquid-filled rate are $35 \%$ and $75 \%$ under braking condition. As the picture shows, the motive direction within the flow channel is almost the same and the speed is spreaded regularly. Owing to the centrifugal force, the flow velocity within the flow channel is proportional to radius, reaching maximum at the outer ring export. When the fast running fluid which split from the export of the pump hits the flow channel of the turbine, it becomes the fluid flows in the turbine flow channel. Because of the inertia force, the liquid first flows within the outer ring of the turbine. But the static turbine blades keep the liquid from continuous flowing and change its motive direction, which lead 
the liquid flows downward and finally out of the turbine flow channel. The flow speed at the center of the turbine flow channel is relatively slow. Comparing the fluid flow within the turbine and pump, the situation in the turbine is more complicated. It includes the irregular phenomena of whirlpool, refluence, secondary flow and so on, which are easily to exacerbate the flow and accelerate the lost of flow in the flow channel. At this time turbine brakes and all the energy transforms to heat energy. Comparing figure ( a) and figure ( $b$ ), it shows that different liquid filled rate has great influence on fluid velocity. Under small liquid filled rate, the scope of low velocity is larger.

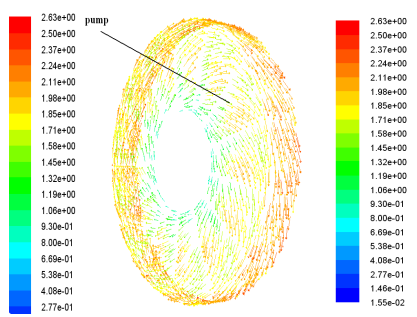

(a) $q_{c}=35 \%$

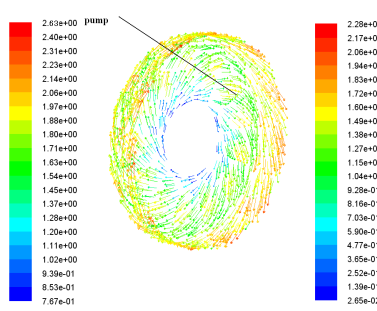

(b) $q_{c}=75 \%$

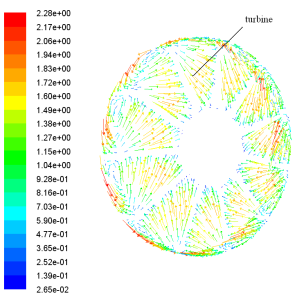

Figure2. contours of velocity vectors

\section{Conclusion and outlook}

Combining the two-dimensional PIV technique and the CFD numerical simulation method, this thesis researchs the gas-liquid two phase flow within the fluid coupling and gets the preliminary reaearch result. It also makes a deep study on the flow characteristic within the flow channle at different liquid filled ratio based on the particle fluid images measured by the PIV test and the flow field velocity, pressure and gas-phase volume distribution images measured by the CFD simulation. According to these research fruits, we can predict the performance of the fluid couplings and factors which affects the performance of it more accurately.

In this experiment, two-dimensional PIV technique is used and can only show the two-dimensional spatial displacement of particles in the flow field. In order to reveal the complex spatial structure within the flow field better and reflect the flow mechanism of the flow field more profoundly, to reflect the flow field properties essentially, the three-dimensional PIV technique research is urgent to be conducted.

\section{References}

[1] Hayami H, Aramaki S , and Watanabe Y, (1997) PC-PIV System for a Measurement of Relative Flow in a Rotating Impeller.The Second International Workshop on PIV'97-Fukui, Japan, July, The Visualization Society of Japan, pp, 105 108.

[2] Sinha M., Katz, J., and Meneveau, C.,(2000) Quantitative Visualization of the Flow in a Centrifugal Pump With Diffuser Vanes-I: On Flow Structurees and Turbulence, ASME J. Fluids Eng., 122,pp.97 107.

[3] k. Sakakibara, M. Yamada, Y. Miyamoto, T. Saito,(2007) Measurement of the surrounding liquid motion of a single rising bubble using a Dual-Camera PIV system. Flow Measurement and Instrumentation, 18, pp. 211 215

[4] Fujiwara A, Minato D, Hishida K.(2004) Effect of bubble diameter on modification of turbulence in an upward pipe flow. International Journal of Heat and Fluid Flow, 25, pp.481 488

[5] Lindken R, Merzkirch W. (2002) A novel PIV technique for measurement in multiphase flows and its application to two-phase bubbly flows. Experiments in Fluids, 33,pp.814 825 